\title{
A EPIDEMIOLOGIA PARA O ENFERMEIRO
}

\author{
Daisy Leslie Steagall Gomes*
}

Este trabalho destaca o uso da epidemiologia pelo enfermeiro com um instrumento indispensável à prestação de serviços de assistência à saúde e sua aplicação na prática profissional. Evidencia igualmente que a disciplina oferece subsídios importantes para o planejamento, administração, execução e avaliação da prestação de serviços à saúde, sendo ainda um instrumento extremamente útil na investigação, o que a torna obrigatória na formação desse profissional.

UNITERMOS: Epidemiologia, vigilância epidemiológica, educação em enfermagem, enfermagem

\section{Introdução}

A Epidemiologia aplica-se ao estudo de todas as condições que afetam ou se relacionam com a situação de saúde de uma população, incluindo-se aí, entre outras: a ocorrência de doenças de um modo geral (morbidade, mortalidade, incapacidade); o estudo de relações causais; a distribuição, qualidade e adequação dos serviços de saúde; a nível administrativo, a supervisão, a avaliação e a vigilância do processo saúde-doença; pesquisas clínicas; testes terapêuticos e outros.

O método epidemiológico é uma ferramenta de grande utilidade para sanitaristas, pesquisadores, médicos clínicos, enfermeiros e todas as demais categorias que trabalham

*Professor Associado do Departamento de Enfermagem Materno-Infantil e Saúde Pública da Escola de Enfermagem de Ribeirão Preto da Universidade de São Paulo. 
na área da saúde.

Por isso mesmo, a formação em epidemiologia constitui assim um dos pilares fundamentais na formação de recursos humanos na saúde, pois ela é o instrumento que permitirá: conhecer a situação de saúde da comunidade; conhecer os fatores causais determinantes do mecanismo de produção das enfermidades; identificar os grupos mais sujeitos aos riscos e as áreas prioritárias de ação; orientar e colaborar no planejamento e na adoção de decisões; colaborar e participar na avaliação do processo de controle das enfermidades e gerar conhecimento facilitando a compreensão de saúde como um todo e conhecer o contexto no qual se geram e explicam os fenômenos de saúde.

A epidemiologia, em seu percurso histórico, caminhou desde a visão e interpretação do mágico ao biológico, do unicausal ao multicausal, ao ecológico e ao histórico-social. As mudanças nas teorias e nos métodos de controle de enfermidades que foram utilizadas em décadas anteriores mostram-se ainda insuficientes para responder à situação atual, daí a busca de teorias mais amplas para descrever e explicar as circunstâncias que contribuem para certos estados de saúde.

Encontram-se, nos autores, definições para os determinados e suas relações com o processo saúde/doença, na visão conceitual de cada um. Alguns autores atribuem as relações aos determinantes biológicos, ficando aos de natureza sócio-econômica e política um desempenho secundário ou complementar.

Autores como MAC MAHON, 1975; LEAVEL, CLARK, 1976; FORATTINI, 1976; ROJAS, 1978 entre outros, definem epidemiologia de modo bastante semelhante, tendo como ponto comum "o estudo da distribuição das doenças nas coletividades humanas e dos fatores causais responsáveis por essa distribuição" (ROUQUAYROL et al. 1988).

Este conceito toma por base as relações existentes entre os fatores do ambiente, do agente e do hospedeiro ou suscetível. Este conceito engloba desde as características demográficas pessoais - idade, sexo, cor até comportamentos e hábitos, como o de fumar, por exemplo. O econômico e o social são partes tão importantes quanto os demais elementos ambientais na gênese das doenças que incidem sobre coletividades humanas.

Outros autores como LAURELL, 1976; AROUCA, 1976; BREIHL, 1980; RUFFINO NETO, PEREIRA, 1982 E CARVALHEIRO, 1986, avançam, no dizer de ROUQUAYROL et al., 1988, em direção a uma nova epidemiologia cuja visão dialética se posiciona contra a fatalidade do "natural" e do "tropical". Nessa nova epidemiologia, também chamada de epidemiologia social, o enfoque dado segundo BREIHL é uma linha de crítica permanente ao capitalismo por considerá-lo patógeno e, portanto oposto por natureza à prática de uma epidemiologia eficaz em benefício da comunidade.

A epidemiologia social permite ir além do problema de saúde específico, para considerar a sociedade como fonte de explicação para os problemas e portanto remeter as 
soluções para a própria sociedade, que deverá ser mobilizada com a finalidade de conseguir gerar soluções ou modos de intervenção no processo.

Os modelos vêm sendo substituídos à medida que novos conhecimentos são produzidos e estes evidenciam a existência de relações mais complexas.

Para MAZZAFERRO, 1987, o paradigma do processo saúde-doença e das políticas de saúde, teve mudanças com o passar dos anos, desde o mágico (aceitação do sobrenatural), passando pelo biológico (descobrimento do germe e a teoria unicausal), o multicausal, o ecológico, o histórico-social (materialista: saúde como qualidade social, estreita relação com a conduta social e estilo de vida) até o político determinativo: política que contempla os fatores herdados, ambientais, biológicos e sócio-econômico-cultural (integrador).

Este último, o integrador, mostra que a análise funcional tradicional pode-se contemplar com o método dialético, com a finalidade de se aprofundar no conhecimento.

Alguns autores acreditam que se possa considerar a descrição funcional e interpretála pelo marco teórico dialético. Não haveria incompatibilidade em definir os fatos que ocorrem com uma visão funcionalista e interpretá-la dialeticamente (CARVALHEIRO, 1984).

Como assinalou BARBOSA, 1984, "A epidemiologia é uma só, apesar de que didaticamente, se possa considerar uma postura clássica e outra social. As duas completam-se; uma analisando o macro universo das relações saúde-doença, e outra dissecando os microelementos dessas mesmas relações. Ambas têm contribuído de modo substancial no esclarecimento dos determinantes do binômio saúde-doença".

Por representar a epidemiologia o instrumento principal para a saúde pública a fim de indicar as medidas de prevenção e de controle a serem adotadas dentro dos recursos disponíveis e dos objetivos a serem atingidos, é proposta deste trabalho destacar a importância de seu ensino para os cursos de formação do enfermeiro.

\section{A epidemiologia para o enfermeiro}

Entre as mudanças que se fazem necessárias para o apoio da enfermagem ao projeto de oferecer a toda a comunidade a assistência à sua saúde, está o seu preparo, quer a nível de formação básica, através de uma educação continuada, para poder coordenar as ações de saúde, dentro de nova estruturação dos serviços que se implantam no país e, como conseqüência, novas funções ou ampliações das já existentes.

O trabalho de enfermagem tem sua especialidade e, através da assistência que presta, contribui para a integralidade da assistência à saúde. 
Nessa assistência identificam-se procedimentos, tarefas e atividades que as categorias vêm realizando na prática e que podem ser expressas por ações.

Este conjunto de ações que caracteriza o trabalho de enfermagem nas instituições de saúde permite destacar dois aspectos importantes: um ligado à essência, o serviço oferecido à clientela; e o segundo, correspondente ao apoio e à qualificação da assistência prestada.

No primeiro, realização de ações propedêuticas e terapêuticas complementares e da enfermagem e as de controle de risco. No segundo, as de natureza administrativa e pedagógica que viabilizam o desenvolvimento das ações anteriores de forma integral, oportuna e com diminuição de risco (ABEn, 1989).

A assistência de enfermagem pode ser conceituada como um conjunto de ações de naturezas diversas que se articulam e se complementam entre si na consecução da finalidade do trabalho em saúde.

Especificamente na vigilância epidemiológica, as ações exercidas por este profissional têm sido de: participar na ordenação dos dados, na produção de novas informações, propor novas metodologias para sua obtenção (estudos especiais, investigações epidemiológicas); realizar análise das limitações, selecionar e aplicar as metodologias mais adequadas para o alcance dos objetivos propostos pelo programa e que sejam mais adequados ao conhecimento das doenças e sua evolução; participar na seleção de alternativas e prioridades e colaborar na elaboração e execução dos programas de controle, assim como avaliação do alcance dos objetivos propostos.

A comunicação e a informação permeiam todo o processo da vigilância epidemiológica, em todos os níveis de ação (local, estadual e nacional) e são básicas na dinâmica dos serviços. A vigilância epidemiológica pode ser resumida como a informação para a ação (STEAGALL-GOMES, MENDES, 1992).

Ainda que várias disciplinas possam trazer contribuições valiosas para a assistência de enfermagem a indivíduos ou grupos de indivíduos, a epidemiologia se destaca pelo seu emprego e/ou utilização para planejar, implementar e avaliar o cuidado à saúde para populações referidas, bem como para instrumentar a realização de pesquisas.

O enfermeiro na sua prática trabalha rotineiramente com dados que são coletados e compilados para registros e relatórios. No entanto, do ponto de vista da administração, esta "contabilidade"** nem sempre apresenta consistência necessária para sua apropriada utilização, uma vez que não são indicadores reais de serviço.

Se, no entanto, esses dados coletados forem relacionados à população atendida por aquele serviço e submetidos a uma análise epidemiológica, poderão permitir identificar a

\footnotetext{
${ }^{\star *}$ Contabilidade: atribuiu-se este nome pelo fato de serem números que não são coeficientes ou taxas
} 
taxa da utilização dessa unidade de saúde; determinar e identificar problemas de saúde já contemplados pelos serviços e até aqueles que ainda não estão sendo atendidos e que merecem ser considerados.

Do ponto de vista administrativo, tais análises podem se voltar para um núcleo extremamente importante, que é a eficácia dos serviços de saúde.

O ensino da epidemiologia para o enfermeiro daria a ele oportunidade de utilizá-lo como um instrumento de planejamento da programação, acompanhamento e sua avaliação.

Torna-se uma disciplina básica por se constituir uma forma de pensar e entender os problemas e as ações de saúde, onde se identifica um conjunto de determinantes biológicos, ecológicos, culturais, econômicos, psicológicos e sociais em torno da explicação do fenômeno saúde/doença na população.

Este processo ensino-aprendizagem em epidemilogia na formação acadêmica do enfermeiro não tem como proposta formar epidemiologista, mas o de oferecer durante o seu curso regular, um instrumental básico que lhe permitirá atuar e intervir nos serviços de saúde com mais segurança.

Além de desenvolver o espírito crítico e a capacidade analítica na forma de ver e pensar as situações de saúde, a epidemiologia vai realizar investigações, respondendo as questões que emergem no exercício de sua prática.

Pode-se definir a investigação como o "estudo sistemático de um problema, com o fim de descobrir uma solução para o mesmo e produzir novo conhecimento" (HALL, WEAVER, 1990).

Considerando o aspecto global do trabalho do enfermeiro, e em particular as suas ações na vigilância epidemiológica e a atuação na administração de serviços ou de assistência de serviços ou de assistência de enfermagem, a epidemiologia terá um importante papel nestes processos, não somente nos aspectos de prevenção e vigilância das enfermidades ou ocorrências, mas também na avaliação e análise do impacto de suas ações. Servirá para identificar necessidades, riscos, definir prioridades, melhorar a utilização de recursos entre outros.

Com relação a investigação, sabe-se das limitações enfrentadas para o exercício desta pelos enfermeiros de serviço, mas uma estratégia viável seria a de que se começasse pela análise dos dados de que se dispõe e que são obtidos no próprio serviço. Este exercício de análise levará o profissional a conhecer melhor a situação de saúde, e também contribuirá para melhorar a sistematização dos procedimentos de coleta e de análise de dados, e ainda estimular o desenvolvimento do processo analítico deles. Nesse processo participam não somente enfermeiros, mas outros elementos da enfermagem e funcionários, que estão envolvidos na coleta e produção de informações, que passariam da mesma forma a conhecer os problemas e a importância de suas ações, no desempenho de sua prática. A premissa básica do ensino desse conteúdo é que se deve considerar o sistema de 
saúde vigente e que o processo de ensino se faça integrado, o máximo possível, com o conhecimento da prática dos serviços, buscando-se sempre contribuir para que haja uma transformação ou melhoria gradativa da assistência à população e que se obtenham profissionais comprometidos com os objetivos e as propostas dos programas e metas da saúde pública.

Deve buscar-se, através da estratégia metodológica do ensino, melhorar a prática dos enfermeiros nessa área, oferecendo-lhes um curso cujo conteúdo teórico e prático os capacite para atender às necessidades de saúde da população (ou melhor, do processo saúde/doença).

A teoria visará a oferecer o instrumento básico para o desenvolvimento da prática, e proporcionar ao aluno a indução à análise crítica do que lhe é oferecido.

O ensino da epidemiologia , como refere MAZZAFERRO, 1987, "deverá estar dirigido de modo que permita acesso a problemática sanitária e aprofundar no conhecimento da cosmovisão integrada da saúde através do aprendizado teórico-prático dos princípios e métodos epidemiológicos".

\section{Considerações finais}

O ensino de epidemiologia desenvolve habilidades para estabelecer prioridades dentro dos limites dos recursos existentes, sensibilizar e organizar a participação da comunidade na área da saúde, estabelecer metas, organizar programas, realizar investigações e avaliações. Possibilita identificar, analisar e interpretar tendências, determinantes políticos, sociais e econômicos e operar interferências para a solução de problemas.

A epidemiologia contribui como instrumento de investigação que possibilita: adequar recursos e serviços para atender às necessidades de saúde e seus determinantes; realizar a comunicação objetiva entre profissionais da administração e os da saúde; capacitar o enfermeiro para suas ações nas atividades junto a vigilância epidemiológica.

A epidemiologia se configura assim num instrumento valioso para utilização dos enfermeiros em sua prática, que seja no ensino, nos serviços, nas suas atividades administrativas específicas e nas investigações.

Seu conteúdo deverá ser oferecido ao enfermeiro, por ser considerado um conhecimento necessário para sua prática profissional, tornando as ações de enfermagem mais efetivas e conscientes.

"Como tudo isso, a epidemiologia é uma boa ferramenta, cabendo-nos usá-la da melhor forma, conscientes dos seus limites, aperfeiçoando-a no que for possível" (ALMEIDA FILHO, 1989). 
The subject of this paper emphasizes the uses of Epidemiology to nurses like an essential tool to afford health service assistance and his application in practice.

Equally it evidences that epidemiology offers important support to the planning, administration, execution and evaluation of the health service and in addition an extremely useful and essential investigation instrument to his professional formation.

UNITERMS: Epidemiology, population surveillance, education nursing, nursing

Este trabajo resalta la utilización de la epidemiología por el enfermero como un instrumento indispensable a la prestación de servicios de asistencia a la salud u su aplicación en la práctica profesional. Evidencia similarmente que la disciplina ofrece subsidios importantes para el planeamiento, administración, ejecución y avaluación de la prestación de servicios a la salud, siendo todavía un procedimiento extremamente útil en la investigación, lo que torna obligatorio en la formación de ese profesional.

UNITERMOS: Epidemiología, vigilancia epidemiológica, educación en Enfermería, Enfermería

\section{REFERÊNCIAS BIBLIOGRÁFICAS}

01. ALMEIDA FILHO, N. Epidemiologia sem números: uma introdução crítica a ciência epidemiológica. Rio de Janeiro, Campus, 1989. 108 p.

02. AROUCA, A. S. S. O Dilema preventivista. São Pualo, 1975. 261 p. (Doutorado). Universidade de Campinas.

03. BARBOSA, F. S. A epidemiologia como instrumento de análise. Senecta, Rio de Janeiro, v. 7, n. 2. P. 31-5, 1984.

04. BREIHL, P. L. Producción y Distribuición de la salud/enfermedad como hecho coletivo. Quito, Editorial Universitário, 1980. 360 p. 
05. CARVAlHeiRO, J. R. Processo migratório e disseminação de doenças. Textos de apoio - Ciências Sociais I, Rio de Janeiro, PEC/ENSAP, ABRASCO, 1983.

06. - Perspectivas de la investigación epidemiológica aplicada a la valuación de la salud. In: USOS y perspectivas de la epidemiologia. Washington: OPS/OMS, 984. p. 133-40. (PNSP 84-47).

07. FORATINI, O. P. Epidemiologia geral. São Paulo, Edgard Blucher, 1976. 259 p.

08. HALL, J. E., WEAVER, B. R. Enfermería en salud comunitária: um enfoque de sistemas. 2. ed., Washington: OPS/OMS, 1990.

09. LAURELL, A. C. A saúde e a doença como processo social. In: Barata, R. C. B. A historicidade do conceito de causa. Textos de apoio: Epidemiologia. Rio de Janeiro. ABRASCO, 1985, p. 13-27.

10. LEAVEL, H. CLARK, E. G. Medicina Preventiva. São Paulo: Mc Graw-Hill, 1976. 744 p.

11. MAC MAHON, B. PUGH, T. F. Princípios y métodos de epidemiologia. 2. ed., México, Prensa Médica Mexicana, 1975, 339 p.

12. MAZZAFERRO, V. E. Epidemiologia como conhecimento básico. In: LA FORMACIÓN en epidemiologia para el desarrollo de los servicios de salud. Washigton: OPS, 1987. p. 59-65 (Serie Desarrollo de Recursos Humanos, 88).

13. ROJAS, R. A. Epidemiologia. Buenos Aires. Intermédica, 1974, v. 2.

14. ROUQUAYROL, M. Z. et al. Epidemiologia \& Saúde. 3. ed. Rio de Janeiro, MEDSI, 1988. $516 \mathrm{p}$.

15. RUFFINO NETO, A., PEREIRA, J. C. O processos saúde-doença e suas interpretações. Medicina, Ribeirão Preto, v. 15, n. 1-2, p. 1-4, 1982. 
16. SEMINÁRIO NACIONAL PARA DEFINIÇÃO DA POLÍTICA E DIRETRIZES PARA A PROFISSIONALIZAÇÃO DO PESSOAL DE ENFERMAGEM, 1989, Brasília. Relatório. Brasília: ABEn, 1989.

17. STEGALL-GOMES, D. L. MENDES, I. J. M. A comunicação e a informação na vigilância epidemiológica. In: SIMPÓSIO BRASILEIRO DE COMUNICAÇÃO EM ENFERMAGEM, 3., 1992, Ribeirão Preto: Anais. Ribeirão Preto: EERP/USP, 1992. p. 366-72. 